tenacious, elastic, and insoluble skin. Such a skin is, I think, admirably adapted to the arrest of hæmorrhagic oozing. I have employed it for this purpose with very satisfactory results, painting it upon the gums in a case of hæmophilia where bleeding had persisted for weeks in spite of the local application of adrenalin chloride and ferric chloride.

The formalin gelatin finds, however, its most generally useful application when it furnishes a protective antiseptic skin to denuded surfaces. It furnishes here an antiseptic and insoluble coating which protects the underlying tissues and is not removed by ordinary washing.

Again, inasmuch as pyogenic micro-organisms (staphylococci) have in the experiments I have carried out been rapidly devitalised when imprisoned under a skin of formalin gelatin, it would seem possible that this material may render services to the operating surgeon. Painted upon the skin it might conceivably furnish the aseptic surface which is required. Conceivably the surgeon might carry his incisions through such a film. Again formalin gelatin might prove useful in sealing up operation wounds, especially operation wounds of mucous surfaces.

Where employing the formalin gelatin upon a denuded surface, pain is avoided by applying first a film of simple gelatin and coating this over when dry with the formalin gelatin. When proceeding thus a tube of sterilised gelatin is melted in hot water and applied to the wound with a brush. This done a volume of formalin equivalent to a twentieth of the bulk is added to the remaining liquid gelatin. The 2 per cent. formalin gelatin thus formed must be applied before it has time to set.

3. On a trypanosoma-like organism found in association vith some chronic pathological affections of the mouth.-The attention of many pathological observers being for the moment focussed upon the study of the life-history and pathological significance of trypanosomata and allied forms it is perhaps opportune to point out that suitable material for the investication of these problems may perhaps lie ready to hand. I have in view pyorrhoea alveolaris and other chronic affections of the month and the micro-organisms which have been described by French bacteriologists, and in particular by Vincent, under the names of bacillus fusiformis (or bastilis) and spirillum buccæ. The micro-organism denoted by the name of bacillus fusiformis is, I think, clearly not of bacillary nature. Judging from its size (ranging sometimes up to $20 \mu$ ), its staining reactions with Leishman's stain (blue staining of the protoplasm and development of three or four chromatin bodies stained red in a characteristic manner), and from the fact that it moves actively through a hanging-drop preparation with an eel-like undulatory morement, the micro-organism would, in spite of the apparent absence of undulatory membrane and fligellum, appear to have affinities with a trypanosome. The resemblance to a trypanosome is heightened when the organism is seen ploughing its way through the corpuscles of a drop of added blood. The micro-organism denoted by the name of the spirillum bucce is practically always found in association with the above. I have, however, failed to find it in one of three cases of chronic mouth affections in which the so-called bacillus fusiformis has been present in large numbers. The spirillum buccæ as seen under the microscope in a moist film executes incessant whip-like movements which bring up vividly to mind the lashings of the flagellate body in malarial blood. The spirilla here in question do not take the stain deeply when treated with watery solutions of basic aniline dyes.

It may be stated that neither of the above-mentioned forms of micro-organisms has as yet been artificially cultivated.

It has been suggested with regard to them that they may be modifications of, or different stages of, one and the same type of micro-organism. This suggestion falls in with the fact that in one of the cases of pyorrhoea alveolaris which I have had under examination there were to be seen many forms transitional between the thicker and shorter form denoted the bacillus fusiformis and the thinner and longer form denoted the spirillum buccæ. It is hardly necessary to point out that the suggestion of a common origin for the two forms acquires particular interest in connexion with the suggestion of Schaudinn that there may be a trypanosoma stage in the life-history of many pathogenic spirilla.

'I'wo further points may be briefly alluded to. (a) The first applies to a case where the pathological affection of the mouth takes the form of a chronic herpetiform eruption on the lips and the development of plaques upon the inner surface of the cheek in the neighbourhood of the angle of the mouth and elsewhere. Here felted masses of the so-called bacillus fusiformis are found in preparations made by puncturing the plaques. 'This is of interest in connexion with the fact that the development of plaques is a characteristic feature in dourine, a form of trypanosoma invasion which affects in particular the genital system of the horse. (b) A condition of extreme anæmia which suggests a possible blood infection is often associated with pyorrhcea alveolaris. In the two cases I have had under observation I have in each case examined the blood films for parasites with negative results.

In conclusion, I desire to express my thanks and obligation to Captain S. R. Douglas, I.M.S., for valuable help and suggestion in connexion with the investigation of these cases. Low er Seymour-street, W.

\section{THE TREATMENT OF CONGENITAL EQUINO-VARUS DURING EARLY INFANCY.}

\section{By E. LAMING EVANS, M.A., M.D. Cantab,} F.R.C.S. ENG.,

STRGEON TO THF ROYAL ORTHOPADIC HOSPITAL.

THE question of the treatment of congenital equino-varus during early infancy is not only of supreme importarce to the patient but also of great interest to the surgeon because of the difficulty in obtaining consistently goor results and of the divergence of opinion as to the methods to be adopted. Indeed, few surgical subjects of such antiquity possess so many different principles and multifarious practices at the present day; neither can it be claimed that the literature upon the subject is not full and exhaustive. It is this ambiguity upon the subject that leads to the number of incomplete corrections that present themselves to our hospitals and induces me to record my experience during the year 1903. During that period 53 cases of congenital equino-varus were treated by me. Two cases were so slight that they were treated solely by manipulation. Of the remaining 51 cases, 40 were of the nature of relapsed cases or incomplete corrections, or were primary cases neglected during early infancy. These were all treated as in-patients, some by tenotomy, fasciotomy, syndesmotomy, and manipulation; others by the more severe methods of tarsal osteectomy or subcartilaginous enucleation. The remaining 11 cases presented themselves during early infancy and were treated as out-patients. They represented varying severities of the so-called second degree; in no case could the inversion, adduction, or plantar flexion be temporarily corrected by manipulation, however forcible. The age varied from two weeks to six months, averaging eight weeks. Nine cases were males and two were females. 14 feet were affected, the right foot in nine cases and the left foot in five.

The method of treatment adopted in all these cases was a slight modification of that first introduced by Dr. W. T. Little and afterwards emphasised by $\mathrm{Mr}$. W. Adams. It is usually ascribed to the latter on the continent. It is an amalgamation of the principles of the mechanical method of Scarpa with the practice of subcutaneous tenotomy of Stromeyer. It consists of two stages: (1) the correction of the varus and (2) the correction of the equinus. Stage 1 is commenced as early as possible, in this anticipating the original operation by a few months. It consists of the subcutaneous division of the tendons of the tibiales anticus and posticus and flexor longus digitorum above the ankle-joint with immediate rectification of the varus so far as possible with subsequent manipulation to complete the correction, and in the intervals of manipulation the retention of the foot in the improved position by means of a padded malleable-iron splint. Manipulation at this stage is directed entirely to the correction and over-correction of the varus at the mid. tarsal joint. So long as, at this stage, the tendo Achillis remains taut, all movements in the ankle and astragalo calcaneal joints are prevented, and we are at a mechanical advantage in overcoming the adduction and inversion at the mid-tarsal joints by being able to manipulate the fore part of the foot upon, as it were, a solid bar of bone extending from that joint to the knee. 'The method of manipulation 
cannot be better or more tersely described than in the words of Hippocrates himself when dealing with club-foot: "In a word, as if moulding a wax model, you must bring to their natural position the parts that were abnornally displaced and contracter together, so rectifying them with your hands and with the bandaging in like manner as to bring them into their natural position, not by force, but gently." The time taken to correct the varus averaged in my series 30 days.

The main advantages of a malleable-iron splint over plaster-of-Paris are : (1) manipulation can be carried out at home in the intervals of attending the surgeon; (2) if the bandage or the splint are soiled by urine they can be taken off, dried, and reapplied or a second set can be immeliately substituted; (3) the mechanical advantage is greater ; and (4) the risk of ulceration of the skin is diminished. No ulceration or excoriation occurred in my series.

The second stage is then commenced without delay by subcutaneous division of the tendo Achillis with immediate rectification as far as possible and subsequent manipulation to complete the correction of the equinus, with retention of the foot in the intervals in a padder tin shoe of special design. This shoe has to be worn day and night and is only taken off for manipulation of the foot. It consists of a foot- and leg-piece firmly connected together, the foot-piece being set so as slightly to evert and to dorsi-flex the foot. By this shoe the foot is kept in an over-corrected position until the child begins to walk. Manipulation at the ankle is carried out at a great mechanical disadvantage, for the length of the fulcrum measures only from behind the mid-tarsal joint to the centre of the ankle. Care must be taken that an apparent correction of the equinus is not obtained by an over-extension at the mid-tarsal joint. This is liable to occur unless the anterior limit of the fulcrum is persistently borne in mind. The time occupied in correcting the equinus varies more than that of the varus and in my experience is usually longer. It is well at this stage to commence to correct any internal rotation of the leg or thigh by manipulation.

The results of the treatment may be recorded thus: feet with full correction, nine; feet with incomplete correction of the equinus, two; foot with incomplete correction of the equino-varus, one. The deformity in this case is associated with a condition of Mongolian imbecility. Two cases cannot be recorded, as one has left London and the other is in a fever hospital. The full correction of the internal rotation of the whole limb is still incomplete in all these cases.

A cure is not claimed in any case. The maximum effect of treatment at this stage is correction of the deformitv. Relapse is constantly tending to occur in all and certainly will in some unless means are adopted to prevent that tendency. These means consist of a short daily manipulation, aided in the majority of cases, and in practically all hospital cases, by some form of walking apparatus. Hippocrates clearly recognised and insisted that cure by his method of manipulation was not attained till adult life, for he says, "This, then, is the mode of cure and it requires neither cutting, burning, nor any other complex means, for such cases sooner yield to treatment than one would believe. However, they are to be fairly mastered only by time and not until the body has grown up in the natural shape." The time occupied in the correction is shortened since the days of Hippocrates but not that in the cure.

The treatment of congenital equino-varus may be divided into two main schools: (1) those who treat during early infancy, employing various methods; and (2) those who wait till childhood has well advanced and then correct by one of the various major operative procedures. To turn to the latter firs ${ }^{2}$, the arguments used in its favour are the dangers of chloroform during early infancy, the risk of ulceration from pressure of the plaster case, the insufficient support of the latter because it softens with the nrine, the long course of the treatment during which the child suffers pain, and the $p$ rents are much inconvenienced. 'The chloroform argument is twice answered; for, first, the risk in the hands of a skilled anæsthetist is no greater at the age of eight weeks for simple manipulation or tenotomy than it is at the age of six years for an extensive tarsectomy ; and, secondly, in ten cases out of 11 in this series no chloroform at all was used. The plaster-of-Paris argument does not apply to this method as it was not used in any case.

The question of the length of treatment requires full consideration. Undoubtedly by postponing all treatment till, for example, the sixth year and then performing tarsectomy six years of treatment are avoided, but during those six years the child is crippled and deformed and a lasting im. pression is liable to be made upon the developing mind. I know of no serious claim for treatment of congenital equino-varus, except after-treatment, by which relapse can be avoided and I hold that no case can be claimed to be cured until the period when all growth has stopped. It is. true that cases treated by tarsectomy at this age with subsequent ankylosis relapse less frequently than those treated by methods which retain free mobility of the tarsal joints, but such cases do relapse and it is impossible to say that any one particular case will not relapse ; hence it is necessary to bestow care and supervision in all cases treated by tarsectomy and after-treatment in those which show a tendency to relapse. In my experience the most troublesome cases that we have to deal with are those which have relapsed after extensive bone sections and obliteration of many joints and I have grave doubts if the time occupied by the treatment of a series of cases at the age of six years by tarsectomy would be materially less than of a series commenced in early infancy, provided the mothers are stimulated by the surgeon to carry out his simple directions with care, perseverance, and enthusiasm.

In the present series three cases have been under treatment for 12 months and the patients have attended the hospital on the average 16 times, and I anticipate their attendance four times a year until the age of six years, thus bringing the total attendances to 36 in six years. In the case of a tarsectomy we must allow a stay in the hospital of at least 18 days. We can thus see that the figures are not altogether so unfavourable to the early treatment. The pain is slight and is inflicted at a time when the memory is undeveloped and impressions are obliterated at once. Indeed, in their treatment these babies often do not cry at all and if they do the cry is far less vigorous than that caused by the stimulus of an empty stomach. 'This argument need scarcely be considered further. This school is of recent date and is tending to grow and to cast into oblivion those apparently tedious and protracted means by which the treatment was carried out with steady advancement during the middle third of the last century. It depends upon the perfection of aseptic methods by which the surgeon can adopt more drastic methods with safety to the patient but, as it appears to me, without due regard to the functional activity and rsthetic appearance of the foot.

Far be it from me to minimise the enormous strides that aseptic surgery has enabled us to accomplish in cases of inveterate club-foot of adolescents. Our knowledge of the morbid anatomy has been increased by opportunities for observation during operative proceedings and our treatment has advanced pari passu with our knowledge of the morbid anatomy; our results are more certain with our increase in knowledge of the technique of aseptic surgery. All these points have combined to diminish the importance of the advantage, if not the necessity, of treatment during early infancy. I maintain that we should reserve such measures for the all too freqnent class of relapsed and neglected cases of adolescence and that we should not be influenced by our success in such cases to slacken our efforts in early infancy.

Turning now to the school that advocates treatment during early infancy the methods are various but usually dogmatic. Thus Erichsen recommends a block-tin splint and manipulation to correct the varus and to convert the compound deformity into simple equinus; then manipulation or tenotomy of the tendo Achillis. In a considerable number of cases, he says, the inversion cannot be corrected until the tense structures which maintain it have been divided. Holding that the ligaments of the inner border of the foot play a more important part than the muscles in maintaining the deformity he recommends Parker's syndesmotomy. Accordingly the division of the tendons of the tibial muscles above the ankle has been replaced by the more efficient method of dividing the tense ligaments in the foot itself and by a single operation bringing the foot into the best possible position. My experience has so far been that a simple division of the tendons of the tibial muscles and the flexor longus digitorum, with immediate rectification of the deformity as far as possible and subsequent manipulation, has sufficed to convert the compound into the simple deformity in an average of 30 days.

Mr. H. H. Clutton, in Treves's "System of Surgery," advocates manipulation and plaster-of-Paris during the first few months of infant life. If the inversion is in great 
measure corrected the tendo Achillis and post ligament of the ankle may alone require division. If deformity is uncorrected when the foot has been brought to a right angle or beyond this point he advises Phelps's operation. $I$ think that if the tibial tendons are clivided at the commencement of treatment part at any rate of the first few months of infant life are spared manipulation and plasterof-Paris and if the division of the tendo Achillis is postponed until the varus is completely and not only in great measure corrected Phelps's operation at so tender an age can also be avoided.

The investigations of Parker and shattock into the morbid anatomy of congenital equino-varus were the first to disturb seriously the ascendancy of $\mathrm{Dr}$. W. J. Little's treatment. Parker advises the division of the tendo Achillis quite early in the treatment of these severe cases, for he says it is impossible properly to gauge the part played by other strnctures so long as the tendo Achillis remains uncut. The old argument, to leave it quite to the last, that it may serve as a point d'appui through which to exert mechanical force on the other structures, is wrong in principle since tenotomy has been introduced. If it prove necessary in any given case it may be taken for granted that the offending structures have not been divided. I have never tried this method in its entirety. I have on a few occasions divided the tendo Achillis before the varus was completely corrected and have found on each occasion the correction of the residual varus troublesome and tedious. I consider that this point d'appui is of the greatest advantage in the correction of the varus, for by it we are enabled to mould the cartilage and to diminish the angle of the neck of the astragalus and to straighten out the incurvation of the anterior extremity of the os calcis, both of which points in the morbid anatomy of congenital club-foot were shown by Parker with geometrical accuracy to be offending structures. But I am not aware that he ever divided or advocated division of these cartilages.

And lastly, if we turn for guidance to one of the most modern text-books of the present day we find that Rose and Carless say, "The tibial tendons are usually first divided and the tendo Achillis subsequently, although some surgeons, and especially Mr. Edmund Owen, recommend that the latter should always be first dealt with as this will occasionally suffice alone." No fairer or terser résumé of the teaching of the present day could be written than the above, but I must own that if I were confronted on some lone Pacitic islanc with a case of congenital club-foot and given those directions I should be at a loss to know whether to nail my flag to the mast of Mr. Owen's always or to the unrecorded authority for the usual practice.

Bryanston-street, W.

\section{AN EXAMINATION OF APPARATUS PRO- POSED FOR THE QUANTITATIVE ADMINISTRATION OF CHLOROFORM.}

\begin{abstract}
By AUGUSTUS D. WALLER, M.D., F.R.S., AND
\end{abstract}

\section{J. H. WELLS, M.R.C.S. ENG., L.R.C.P. LOND.}

\section{I.-INTRODUCTORY.}

THE subjoined contribution to our knowledge of apparatus proposed for the quantitatively regulated administration of chloroform for anæsthetic purposes formed Chapter III. of a laboratory report of "Experiments and Observations relating to the Question of Chloroform Dosage," based upon experiments by Mr. J. H. Wells and by myself, presented to the special chloroform committee of the British Medical Association in July, 1903. It is important that facts and conclusions obtained by independent investigation relating to the question of safe and unsafe methods of effecting anæsthesia shonld not remain unpublished. I give, therefore, in its original form Chapter III. of the report (methods and apparatus for regulated administration). 'The chapter in question forms Section II. of the present paper. My further appreciation of the relative safety of the three forms of apparatus actually recommended in this country is an addition confirmed by further experience. It did not exist in the original report and forms Section III. of the present paper.
II.-QUAN'TTA'TIVE ADMINISTRATION.

The practical recommenclation of the Scotch school of anæsthetists, "to give plenty of chloroform with plenty of air," translated into precise language, becomes an instruction to administer the vapour ad libitum, diluted by from 50 to 100 times its volume of air. The large amount of chloroform used and its free admixture with air produce in effect a large volume of mixture and therefore a more nearly uniform supply to the patient's lungs than when small quantities and a closer method with its necessarily frequent alternations of "off" and "on" are resorted to. The drop method is a first approach towards quantitative administration. We have given considerable attention, therefore, to the definition and measurement of a drop of chloroform. The weight of a drop varies, of course, with the orifice of delivery ; it is therefore easy to unrlerstand the origin of the very divergent weights attributed to a drop of chloroform by English, German, and French authorities. In Germany ${ }^{l}$ an average drop of chloroform is stated by Eschbaum to weigh 38 milligrammes ( $=7 \cdot 6$ cubic centimetres of rapour). In France ${ }^{2}$ a drop of chloroform is stater by Dubois to weigh 66 milligrammes $(=13.2$ cubic centimetres of rapour $)$. Our statement is that from an average pipette, and from a stoppered bottle, the drop of chloroform comes out at 20 and 25 milligrammes (four and five cubic centimetres of vapour), and that from a given pipette a drop of chloroform is a rery constant amount. Thus a drop of $\mathrm{CHCl}_{3}$, the vapour of which is inhaled with an ordinary inspiration of from 400 to 500 cubic centimetres, supplies a 1 per cent. mixture of chloroform and air. At 16 inspirations per minute. 16 drops, or from 0.320 to 0.400 gramme of chloroform at 1 per cent. dilution in air, would be inhaled and absorbed if the inhalation of the vapour were complete and continuous. And on this estimate anæsthesia should be induced in, say, 10 minutes by an absorption of between three and four grammes of chloro. form, or between 2 and $2 \cdot 6$ cubic centimetres (Angliò $=$ from 34 to 44 minims, or less than one drachm). We have regarded this small matter as important enough to deserve careful attention and have therefore made series of weighings of chloroform and of water delivery by various pipettes and bottles. We subjoin a series of weights obtained in grammes per 100 drops of liquirl.

\begin{tabular}{lcccccccccc} 
& $\begin{array}{c}\text { Pipette } \\
\text { A. }\end{array}$ & \multicolumn{2}{c}{$\begin{array}{c}\text { Pipette } \\
\text { B. }\end{array}$} & \multicolumn{2}{c}{$\begin{array}{c}\text { Pipette } \\
\text { C. }\end{array}$} & $\begin{array}{c}\text { Bottle } \\
\text { stoppered. }\end{array}$ & $\begin{array}{c}\text { Bottle } \\
\text { unstop- } \\
\text { pered. }\end{array}$ \\
100 drops of $\mathrm{H}_{2} \mathrm{O}$ & $\ldots$ & $5 \cdot 700$ & $\ldots$ & $4 \cdot 606$ & $\ldots$ & $2 \cdot 723$ & $\ldots$ & $13 \cdot 597$ & $\ldots$ & $7 \cdot 193$ \\
100 drops of $\mathrm{CHCl}_{3}$ & $\ldots$ & $1 \cdot 849$ & $\ldots$ & $1 \cdot 307$ & $\ldots$ & $1 \cdot 275$ & $\ldots$ & $2 \cdot 285$ & $\ldots$ & $2 \cdot 430$
\end{tabular}

Trial of pipettes of various-sized orifices taught us further (1) to adjust the orifice in the blow-pipe so that a pipette

FIG. 1 .

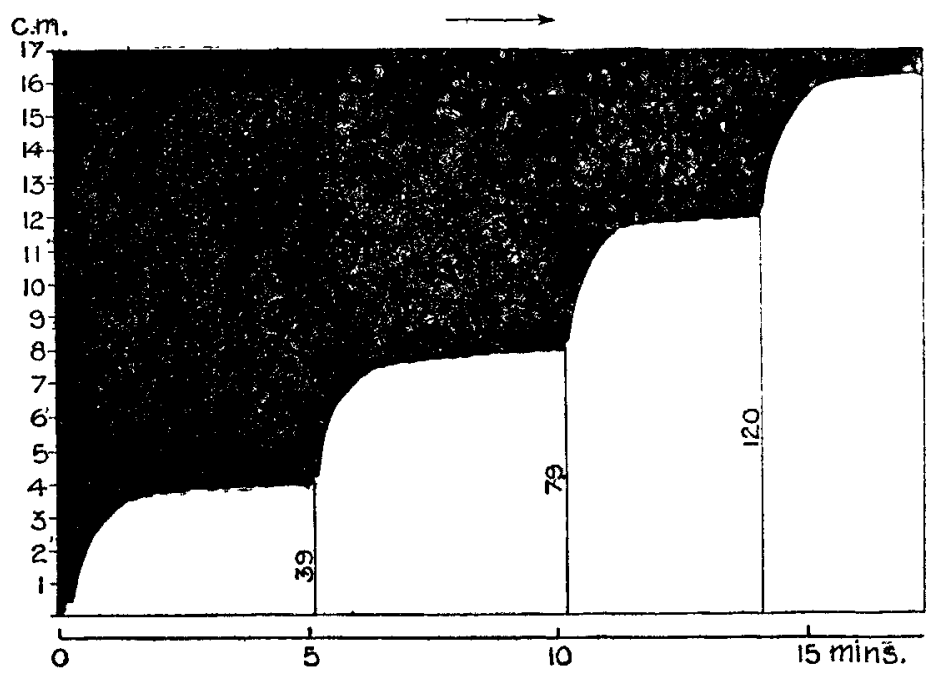

shall deliver as nearly as possible drops of chloroform weighing ten or 15 milligrammes-i.e., hundredths of a gramme or of a cubic centimetre; and (2) to use from such pipettes

1 Eschbaum: Deutsche Medicinische Wochenschrift, No. 23, quoted by Dreser in drchiv fur Pharmäkologie und Pathologie, Band xxxvii., p. 376

2 Dubois: Anesthésie, Paris, 1894, p. 99. The "normal pipette" recently proposed by Yvon (Etude sur le Compte-gouttes Normal Journal de Pharmacie, 1903) has the following dimensions: Interna diameter, from 0.59 to 0.60 millimetre; external diameter, from 3.00 to 3.05 millimetres; and at the pressure of from two to four centimetres of liquid delivers 20 drops of distilled water to one gramme. The chloroform drop from this pipette weighs 0.0165 gramme; the water drop weighs 0.05 gramme. 